\title{
Heavy-Light Decay Constants: Conclusions from the Wilson Action
}

\author{
C. Bernard, ${ }^{*} *$ T. DeGrand, ${ }^{b}$ C. DeTar, Steven Gottlieb, ${ }^{d}$ Urs M. Hellere J. Hetrick ${ }^{\mathrm{f}}$ N. Ishizukag \\ C. McNeile, ${ }^{c}$ R. Sugar, ${ }^{\mathrm{h}}$ D. Toussaint, and M. Wingated \\ a Department of Physics, Washington University, St. Louis, MO 63130, USA \\ ${ }^{\text {b} P h y s i c s ~ D e p a r t m e n t, ~ U n i v e r s i t y ~ o f ~ C o l o r a d o, ~ B o u l d e r, ~ C O ~ 80309, ~ U S A ~}$

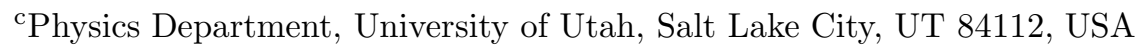 \\ ${ }^{\mathrm{d}}$ Department of Physics, Indiana University, Bloomington, IN 47405, USA \\ eSCRI, Florida State University, Tallahassee, FL 32306-4130, USA \\ ${ }^{\mathrm{f}}$ Department of Physics, University of the Pacific, Stockton, CA 95211, USA \\ 'Institute of Physics, University of Tsukuba, Tsukuba, Ibaraki 305, Japan \\ ${ }^{\mathrm{h}}$ Department of Physics, University of California, Santa Barbara, CA 93106, USA \\ ${ }^{i}$ Department of Physics, University of Arizona, Tucson, AZ 85721, USA \\ ${ }^{j}$ RIKEN BNL Research Center, Upton, New York 11973, USA
}

We report on the results of a MILC collaboration calculation of $f_{B}, f_{B_{s}}, f_{D}, f_{D_{s}}$ and their ratios. We discuss the most important errors in more detail than we have elsewhere.

As is well known, precise computations of heavy-light decay constants such as $f_{B}$ would place stringent constraints on the Standard Model. Reference [1] describes our recently completed evaluation of these decay constant using the Wilson action. We have good control of all sources of error within the quenched approximation. By comparing our quenched results with those from lattices with two flavors of staggered dynamical quarks, we are also able to estimate the error due to quenching. However, for reasons described below, the error on this error is probably rather large.

Rather than repeat the full exposition of Ref. [1], we concentrate only on a few key points here and discuss them in greater detail than was possible previously. This paper should therefore be read in conjunction with [1], which also contains references to related work. Further, to com-

*presented by C. Bernard plement the earlier discussion, we focus on our data for the ratios of decay constants $\left(f_{B_{s}} / f_{B}\right.$ and $\left.f_{D_{s}} / f_{D}\right)$, rather than the decay constants themselves.

The largest error within the quenched approximation comes from (a) the extrapolation to the continuum, in conjunction with (b) the chiral extrapolation, and (c) perturbation theory errors. (Errors (a), (b), and (c) cannot be disentanged with current data.) Figures 1 and 2 show $f_{B_{s}} / f_{B}$ and $f_{D_{s}} / f_{D}$, respectively, as functions of lattice spacing. In each case, two possible extrapolations to the continuum are shown: (1) a linear fit in $a$ to all the quenched data, and (2) a constant fit to all the quenched data with $a \lesssim 0.5 \mathrm{GeV}^{-1}$ $\left(6 / g^{2} \geq 6.0\right)$. Since we expect Wilson fermions to have $\mathcal{O}(a)$ errors, fit (1) is the most natural choice. This choice receives support from the confidence levels of the fits: in both figures, (1) is a much better fit than (2). 


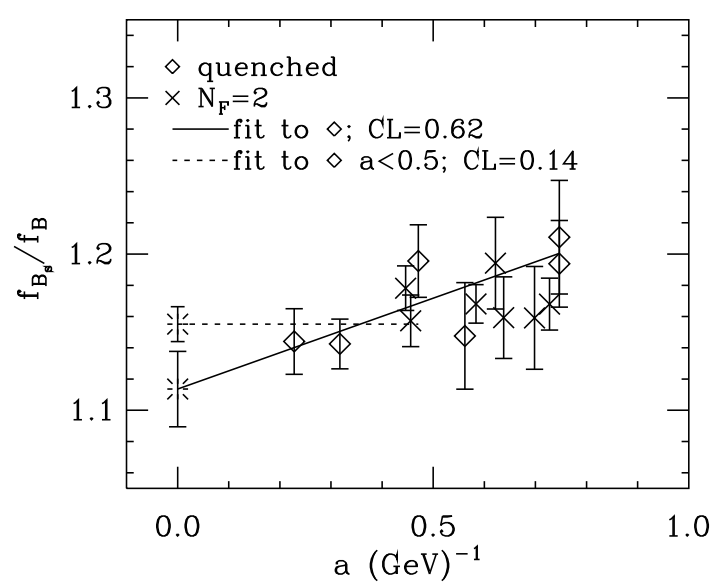

Figure 1. Results for $f_{B_{s}} / f_{B}$ as a function of lattice spacing, with central choices for other elements of the analysis. The fits are to the quenched approximation points (diamonds) only.

For the decay constants themselves, unlike the ratios, the confidence levels of the linear and constant fits described above are both good, with the constant fits in fact having higher confidence levels than the linear fits. (See Fig. 1 in [1].) However it would be inconsistent to treat the decay constants as independent of $a\left(\right.$ for $a \lesssim 0.5 \mathrm{GeV}^{-1}$ ), yet fit the ratios linearly. We therefore choose, for our central values of both decay constants and ratios, the results from the linear fit. Clearly, however, the difference between the fits must be included in the systematic error estimate.

As described in Ref. [1], the errors coming from the chiral extrapolations, as well as perturbative effects beyond one loop, are entangled with the continuum extrapolation error. This is because a change in the types of chiral fits used, or a change in the one-loop scale $q^{*}$, moves the individual, fixed- $a$ points enough to affect significantly the difference between linear and constant continuum extrapolations.

Altogether, we consider 4 choices for the chiral fits and 3 choices for $q^{*}$ (see [1]). We then compute each quantity 24 times ( 2 continuum extrapolations $\times 4$ chiral fits $\times 3$ scale choices), giving a central value and 23 alternatives. The spread in the alternatives (taken separately in the positive and negative directions) determines the combined

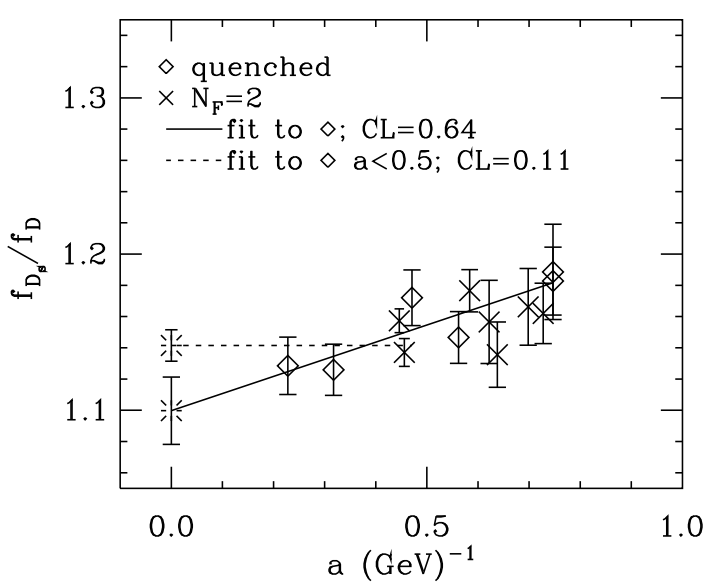

Figure 2.

Same as Fig. 1, but for $f_{D_{s}} / f_{D}$.

systematic error due to these three effects.

For the decay constants, the errors due to each of the three effects alone (determined by variation in the corresponding choices only) are comparable, and the combined error computation is nontrivial. For example, the combined positive error thus determined on $f_{B}$ or $f_{B_{s}}$ is $\sim 25 \%$ smaller than the sum in quadrature of the three individual errors. This is due to the correlations among the errors.

In contrast, for the ratios of decay constants, the errors due to the chiral fits or perturbative corrections alone are quite small. This is illustrated for $f_{B_{s}} / f_{B}$ in Fig. 3 . Changing the chiral fit and/or the $q^{*}$ choice makes very little difference, as long as the linear continuum extrapolation is used in all cases. The combined error for each of the ratios then turns out to be, to a good approximation, simply the average (over all chiral and $q^{*}$ choices) of the difference between the linear and constant continuum extrapolations.

Other sources of error within the quenched approximation are much smaller than the combined error just described and appear to be more or less independent of it. They are added to the combined error in quadrature. See Ref. [1] for details.

We emphasize that the central values quoted come from the quenched approximation. Our $N_{F}=2$ dynamical fermion simulations are used 


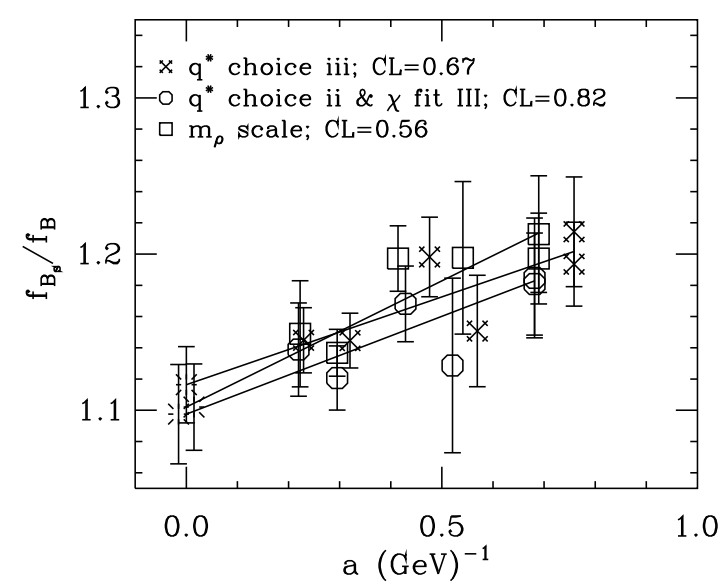

Figure 3.

Quenched $f_{B_{s}} / f_{B}$ with various alternative choices in the analysis.

only to make an estimate of the effects of including virtual quark loops. We feel it would be premature to try quote "full QCD" results because: (1) the virtual quark mass is fixed and not extrapolated to the chiral limit, (2) we do not believe the $N_{F}=2$ data is good enough at this point to attempt an extrapolation to $a=0$, and (3) we have two light flavors, not three.

In practice, the quenching error is estimated primarily by comparing the $N_{F}=2$ results at the smallest available lattice spacing $(a \approx$ $0.45 \mathrm{GeV}^{-1}, \beta=5.6, a m=0.01,16^{3} \times 32$ and $\left.24^{3} \times 64\right)$ with the quenched results interpolated (via the linear fit) to the same lattice spacing. The $24^{3} \times 64 N_{F}=2$ set is new this year and gives decay constants which are consistent with, but somewhat larger than, the decay constants on the older $16^{3} \times 32$ (HEMCGC) set. Since the $N_{F}=2$ decay constants at this lattice spacing are always larger than the corresponding quenched ones (see Fig. 1 of Ref. [1]), we end up quoting a very asymmetric quenching error for $f_{B}, f_{B_{s}}, f_{D}$, and $f_{D_{s}}$. For $f_{B_{s}} / f_{B}$ and $f_{D_{s}} / f_{D}$, the results for $N_{F}=2$ and quenched lattices are consistent within errors (see e.g., Fig. 11). It is not surprising therefore that the quenching error we estimate in this case is therefore roughly the size of the statistical errors.

The effect of changing the way the lattice spac- ing is determined (from fixing $f_{\pi}$ to fixing $m_{\rho}$ ) can also be used as a rough estimate of the size of (some) quenching errors. For the decay constants, this method gives an error much smaller than the direct comparison described above. For $f_{B_{s}} / f_{B}$ and $f_{D_{s}} / f_{D}$, the two approaches give comparable estimates (compare Fig. 3 with Fig. 1). Similar statements apply to the effect of changing the way the strange quark hopping parameter is determined (from fixing the $K$ mass to fixing the $\phi$ mass). We take the final quenching error as the largest error found with any of the methods.

Our results are (in $\mathrm{MeV})$ :

$$
\begin{gathered}
f_{B}=157(11)\left({ }_{-9}^{+25}\right)\left({ }_{-0}^{+23}\right) ; \quad f_{B_{s}}=171(10)\left({ }_{-9}^{+34}\right)\left({ }_{-2}^{+27}\right) \\
f_{D}=192(11)\left({ }_{-8}^{+16}\right)\left({ }_{-0}^{+15}\right) ; \quad f_{D_{s}}=210(9)\left({ }_{-9}^{+25}\right)\left({ }_{-1}^{+17}\right) .
\end{gathered}
$$

The errors are statistical, systematic (within the quenched approximation), and systematic (of quenching), respectively. It must be kept in mind that the error on the quenching error is large. For example, an extrapolation of the $N_{F}=2$ results to the continuum looks like it would significantly increase the (positive) quenching error on the decay constants. Further, even within the current algorithm for estimating quenching, the error on the quenching error is $\gtrsim 50 \%$, due to the statistical fluctuations and the various systematic variations in the analysis.

For the ratios, we find:

$$
\begin{aligned}
f_{B_{s}} / f_{B} & =1.11(2)\left({ }_{-3}^{+4}\right)(3) \\
f_{D_{s}} / f_{D} & =1.10(2)\left({ }_{-2}^{+4}\right)\left({ }_{-3}^{+2}\right) \\
f_{B} / f_{D_{s}} & =0.75(3)\left({ }_{-2}^{+4}\right)\left({ }_{-0}^{+8}\right) \\
f_{B_{s}} / f_{D_{s}} & =0.85(3)\left({ }_{-3}^{+5}\right)\left({ }_{-0}^{+5}\right) .
\end{aligned}
$$

We note that as experimental measurements of $f_{D_{s}}$ improve, the ratios $f_{B} / f_{D_{s}}$ and $f_{B_{s}} / f_{D_{s}}$ may ultimately provide the best way to determine $f_{B}$ and $f_{B_{s}}$.

We thank Y. Kuramashi for disucssions and CCS (ORNL), SDSC, Indiana University, NCSA, PSC, MHPCC, CTC, CHPC (Utah), and Sandia Natl. Lab. for computing resources. This work was supported in part by the DOE and NSF.

\section{REFERENCES}

1. C. Bernard, et al., hep-ph/9806412, submitted to Phys. Rev. Lett. 